\title{
Lumbar Fusion With Polyetheretherketone Rods Use for Patients With Degenerative Disease
}

\author{
Donald A. Ross, MD; and Miner N. Ross, MPH, MD
}

Introduction: Polyetheretherketone (PEEK) rods for lumbar fusion have been available since 2007 . However, literature about their utility is sparse and of mixed outcomes.

Methods: A retrospective review of PEEK rod lumbar fusion cases was performed. Data were analyzed from 108 patients of the senior author Donald Ross who underwent PEEK lumbar fusion.

Results: There were 97 single and 112 -level fusions. Rates of tobacco use, diabetes mellitus, low bone density, depression, and immunosuppression were $23.1 \%, 24.1 \%, 14.8 \%, 32.4 \%$, and $6.5 \%$, respectively. In the study population, the mean age was 60.2 years, body mass index was 30.1 , and there was a mean 31.3 months for follow-up. There were no wound infections or new neurologic deficits. Of 81 patients with $>11$ months of follow-up, $70(86.4 \%)$ had an arthrodesis, $8(9.9 \%)$ had no ar- throdesis, and $3(3.7 \%)$ were indeterminate. No patients had revision fusion surgery and 2 patients had adjacent level fusions at 27 and 60 months. One patient had an adjacent segment laminectomy at 18 months and one a foraminotomy at 89 months, resulting in a $3.7 \%$ adjacent segment surgery rate. Mean preoperative Short Form-36 (SF-36) physical functioning (PF) score and Oswestry Disability Index (ODI) score were 28.9 and 24.8, respectively. Mean SF-36 PF postoperative score at 1 and 2 years were 59.3 and 65 , respectively. Mean ODI postoperative score at 1 year was 14.5 .

Conclusions: In a large patient cohort lumbar fusion with PEEK rods can be undertaken with low complication rates, satisfactory clinical improvements, low rates of hardware failure or need for revision surgery. Longer follow-up is needed to confirm findings.
$\mathrm{S}$ urgical treatment of degenerative lumbar spine disease has been rising steadily in the United States, and an increasing fraction of surgery involves lumbar fusion. ${ }^{1,2}$ Various techniques are used to accomplish a lumbar fusion, including noninstrumented fusion, anterior lumbar interbody fusion (ALIF), lateral lumbar interbody fusion (XLIF, OLIF), posterior pedicle screw fusion, posterior cortical screw fusion, posterior interbody fusion (TLIF, PLIF), and interspinous process fusion. Rigid, metallic fusion hardware provides high stability and fusion rates, but it likely leads to stress shielding and adjacent segment disease. ${ }^{3}$ There is interest in less rigid and dynamic stabilization techniques to reduce the risk of adjacent segment disease, such as polyetheretherketone (PEEK) rods, which have been available since 2007. However, literature regarding PEEK rod utility is sparse and of mixed outcomes. ${ }^{3,4}$ Additional patient reported outcome (PRO) information would be useful to both surgeons and patients. Using institutional data, this review was designed to examine our experience with PEEK rod lumbar fusion and to document PROs.

\section{METHODS}

The study was approved by the institutional review board at the US Department of Veterans Affairs (VA) Portland Health Care System (VAPHCS) in Oregon with a waiver of authorization. In this retrospective, single center study, data were queried from the senior author's (DAR) case logs from VA Computerized Patient Record System (CPRS). Electronic medical records, imaging, and PROs of all consecutive patients undergoing lumbar fusion at 1 or 2 levels with PEEK rods for degenerative disease were retrospectively reviewed. Cases of trauma, malignancy, or infection were excluded. From March 2011 through October 2019, 108 patients underwent lumbar fusion with PEEK rods.

Surgeries were conducted on a Mizuho OSI Jackson Table via bilateral 3 to $4 \mathrm{~cm}$ Wiltse incisions using the Medtronic Quadrant retractor system. Medtronic O-Arm images were acquired and delivered to a Medtronic Stealth Station for navigation of the screws. Monopolar coagulation

Donald Ross is a Staff Surgeon in The Operative Care Division at VA Portland Health Care System in Oregon. Donald Ross is an Attending Surgeon and Miner Ross is a Resident Surgeon, both in the Department of Neurological Surgery, Oregon Health \& Science University in Portland. Correspondence: Donald Ross (rossdo@ohsu.edu)

Fed Pract. 2021;38(suppl 1) Published online April 12, 2021. doi:10.12788/fp.0119
TABLE 1 Patient Demographics ( $N=108)$

\begin{tabular}{|c|c|}
\hline Characteristics & Results \\
\hline Male, No. (\%) & $88(81.5)$ \\
\hline Age, mean, y & 60.2 \\
\hline Current tobacco usage, No. (\%) & $25(23.1)$ \\
\hline Body mass index, mean & 30.1 \\
\hline \multicolumn{2}{|l|}{ Diagnoses, No. (\%) } \\
\hline Diabetes mellitus & $26(24.1)$ \\
\hline Osteoporosis & 16 (14.8) \\
\hline Depression & 35 (32.4) \\
\hline Immunosuppression & $7(6.5)$ \\
\hline
\end{tabular}


FIGURE 1 Anteroposterior and Lateral X-rays

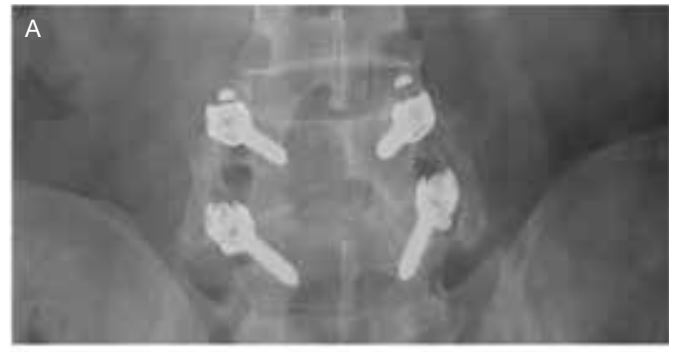

B

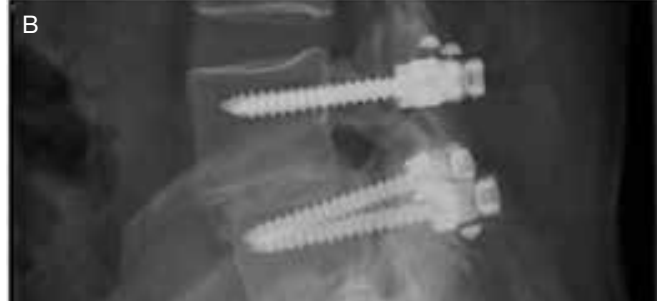

A typical $L 4-5$ fusion construct for spondylolisthesis. The top image shows an anteroposterior polyetheretherketone rods with metal fiducial marker caps at the ends and robust intertransverse fusion bone bilaterally. The bottom image is a lateral view showing the polyetheretherketone rods with metal fiducial marker caps protruding rostral and caudal to the screw heads. The spondylolisthesis is visible at L4-5.

was not used. PEEK pedicle screws were placed and verified with a second O-Arm spin before placing lordotic PEEK rods in the screw heads. No attempt was made to reduce any spondylolisthesis, but distraction was used to open the foramina and indirectly decompress the canal. An interbody device was placed only in treatment of multiply recurrent disc protrusion. After decortication of the transverse processes and facets, intertransverse fusion constructs consisting of calcium hydroxyapatite soaked in autologous bone marrow blood and wrapped in 6-mg bone morphogenetic protein-soaked sponges were placed on the bone. If canal decompression was indicated, a Medtronic Metrx retractor tube was then placed through one of the incisions and decompression carried out. Wounds were closed with absorbable suture. No bracing was used postoperatively. Figure 1 shows a typical single level PEEK rod fusion construct.

Patient pre- and postoperative Short Form36 (SF-36) physical function (PF) scores and Oswestry Disability Index (ODI) scores had been obtained at routine clinic visits.

Static radiographs were used to assess the fusion. Dynamic films and/or computed tomography (CT) scans were obtained only when symptomatic pseudarthrosis was suspected. Some patients had abdominal or lumbar CT
FIGURE 2 Postoperative Sagittal Lumbar Computed Tomography

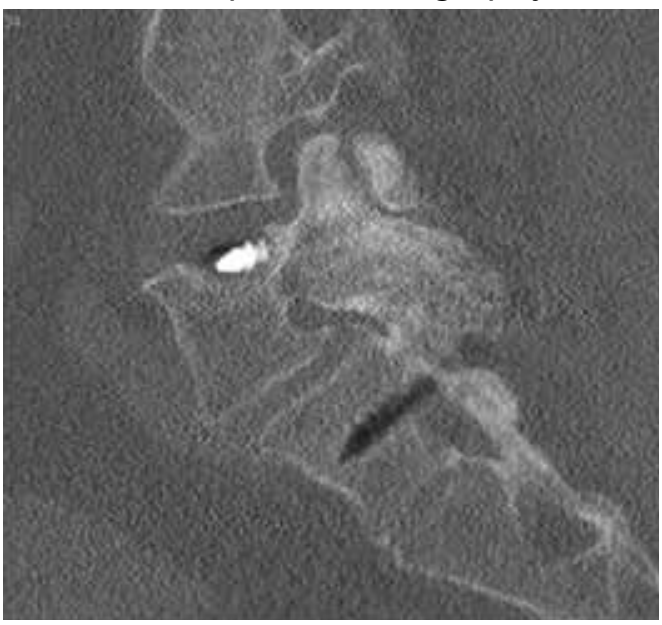

Imaging shows solid facet fusion from polyetheretherketone rod arthrodesis.

scans for other indications, and these were reviewed when available. Particular care was taken to assess facet fusion as an indicator of arthrodesis (Figure 2). ${ }^{5}$

\section{Statistical Analysis}

Pre- and postoperative pairwise $t$ tests were completed for patients with a complete data, using SAS 9.2 statistical package. Data are presented as standard deviation (SD) of the mean.

\section{RESULTS}

Following application of the inclusion/exclusion criteria, 108 patients had undergone lumbar fusion with PEEK rods. Mean (SD) patient age was 60.2 (10.3) years and 88 patients were male (Table 1). Most surgeries were at L5-S1 and L4-5. There were 97 single-level fusions and 11 bilevel fusions. Seventy-four procedures were for spondylolisthesis, 23 for foraminal stenosis, 5 for degenerative disc disease, 3 for coronal imbalance with foraminal stenosis, 2 for pseudarthrosis after surgery elsewhere, and 1 for multiple recurrent disc herniation (Table 2). Twenty-five patients (23.1\%) were current tobacco users and 28 (25.9\%) were former smokers, $26(24.1 \%)$ had diabetes mellitus (DM), 16 (14.8\%) had low bone density by dual energy X-ray absorptiometry (DEXA) imaging, $35(32.4 \%)$ had depression, and 7 (6.5\%) were taking an immunosuppressive agent (chronic steroids, biological response modifiers, or methotrexate). Mean body mass index was 30.1. 
FIGURE 3 Images Showing Arthrodesis
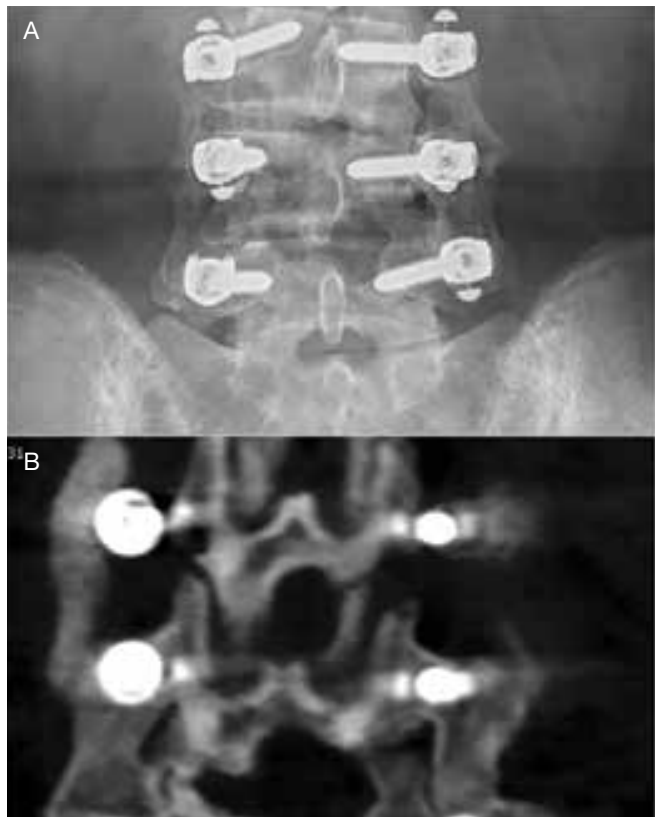

A, anteroposterior lumbar $\mathrm{x}$-ray showing a solid arthrodesis bilaterally at L4-5 and a forming arthrodesis at the more recently operated L3-4 level. B, coronal computed tomography showing a solid arthrodesis bilaterally at L4-5 and a forming arthrodesis at the more recently operated L3-4 level.

\section{Surgical Procedure}

Of the 108 patients, the first 18 underwent a procedure with fluoroscopic guidance and the Medtronic FluoroNav and Stealth Systems. The next 90 patients underwent a procedure with O-Arm intraoperative CT scanning and Stealth frameless stereotactic navigation. The mean (SD) length of stay was 1.7 (1.3) days. There were no wound infections and no new neurologic deficits. Mean (SD) follow up time was $30.3(21.8)$ months.

\section{Imaging}

Final imaging was by radiograph in 73 patients, CT in 31, and magnetic resonance imaging (MRI) in 3 (1 patient had no imaging). Sixtyseven patients $(62.0 \%)$ had a bilateral arthrodesis, and $15(13.9 \%)$ had at least a unilateral arthrodesis. MRI was not used to assess arthrodesis. Eight patients (7.4\%) had no definite arthrodesis. Seventeen patients had inadequate or early imaging from which a fusion determination could not be made. Of 81 patients with $>11$ months of follow up, $58(71.6 \%)$ had a bilateral arthrodesis, $12(14.8 \%)$ had a unilateral arthrodesis, 8 (9.9\%) had no arthrodesis, and $3(3.7 \%)$ were indeterminate.
TABLE 2 Surgical Characteristics

\begin{tabular}{lc} 
Characteristics & Results \\
\hline Reasons for surgery, No. & \\
Spondylolisthesis & 74 \\
Foraminal stenosis & 23 \\
Degenerative disc & 5 \\
Coronal imbalance & 3 \\
Pseudarthrosis & 2 \\
Recurrent herniation of the nucleus & 1 \\
$\quad$ pulposus & \\
\hline Spine level, No. & \\
L5-S1 & 51 \\
L4-5 & 39 \\
L3-4 & 5 \\
L2-3 & 1 \\
L4-S1 & 9 \\
L3-L5 & 3 \\
\hline Length of stay, mean (SD) [range] & $1.7(1.6)$, \\
& {$[0-7]$} \\
\hline Wound infections, No. & 0 \\
\hline New neurologic deficits, No. & 0
\end{tabular}

No patient had any revision fusion surgery at the index level during follow up. Two patients had adjacent level fusions at 27 and 60 months after the index procedure. One patient had a laminectomy at an adjacent segment at 18 months postfusion, and 1 had a foraminotomy at an adjacent segment 89 months post fusion (Figure 3). Overall, there were $4(3.7 \%)$ adjacent segment surgeries at a mean of 48.5 months after surgery. One patient had a sacro-iliac joint fusion below an L5-S1 fusion 17 months prior for persisting pain after the fusion procedure.

\section{Patient Reported Outcomes}

Preoperative SF-36 PF and ODI scores were available for 81 patients (Table 3). Postoperative SF-36 PF scores were obtained at 3 months for 65 of these patients, and at 1 year for 63 patients. Postoperative ODI scores were obtained at 3 months for 65 patients, and at 1 year for 55 patients. Among the 65 patients with completed SF-36 scores at 3 months, a mean increase of $22.4(95 \% \mathrm{Cl}, 17-27 ; P<.001)$ was noted, and for the 63 patients at 1 year a mean increase of $30.3(95 \% \mathrm{Cl}, 25-35 ; P<.001)$ was noted. Among the 65 patients with completed ODI scores at 3 months, a mean decrease of $6.8(95 \% \mathrm{Cl}, 4.9-8.6 ; P<.001)$ was noted, and for the 55 patients with completed ODI scores at 1 year a mean decrease of $10.3(n=55 ; 95 \% \mathrm{Cl}$, 8.4-12.2; $P<.001$ ) was noted. 
TABLE 3 Primary Outcomes

\begin{tabular}{lcc} 
Outcomes & \multicolumn{2}{c}{ Results } \\
\hline $\begin{array}{l}\text { Follow up, mo } \\
\text { Mean, (SD) } \\
\text { Median }\end{array}$ & $29.3(21.5)$ \\
24
\end{tabular}

Abbreviations: ODI, Oswestry Disability Index; SF-36, short form-36

\section{Cost}

We compared the hardware cost of a single level construct consisting of 4 pedicle screws, 4 locking caps, and 2 rods using a PEEK system with that of 2 other titanium construct systems. At VAPHCS, the PEEK system cost was about $71 \%$ of the cost of 2 other titanium construct systems and $62 \%$ of the cost when compared with Medtronic titanium rods.

\section{DISCUSSION}

PEEK is useful for spine and cranial implants. It is inert and fully biocompatible with a modulus of elasticity between that of cortical and cancellous bone, and much lower than that of titanium, and is therefore considered to be semirigid. ${ }^{3,4,6}$ PEEK rods are intermediate in stiffness between titanium rods (110 Gigapascals) and dynamic devices such as the Zimmer Biomet DYNESYS dynamic stabilization system or the Premia Spine TOPS system. ${ }^{3}$ Carbon fiber rods and carbon fiber reinforced PEEK implants are other semirigid rod alternatives. ${ }^{7,8}$ PEEK rods for posterior lumbar fusion surgery were introduced in 2007. Li and colleagues provide a thorough review of the biomechanical properties of PEEK rods. ${ }^{3}$

PEEK is thought to have several advantages when compared with titanium. These advantages include more physiologic load sharing and reduction in stress shielding, improved durability, reduced risk of failure in osteoporotic bone, less wear debris, no change in bone forming environment, and imaging radiolucency. ${ }^{4,9}$ Spinal PEEK cages have been reported to allow more uniform radiation dose distribution compared with metal constructs, an advantage that also may pertain to PEEK rods. ${ }^{10}$ Disadvantages of PEEK rods include an inability to detect rod breakage easily, lack of data on the use in more than minimally unstable clinical situations, and greater expense, although this was not the authors' observation. . $^{3,411}$

Importantly, it has been reported that PEEK rods permit a greater range of motion in all planes when compared with titanium rods. ${ }^{9}$ Polyetheretherketone rods unload the bone screw interface and increased the anterior column load to a more physiologic $75 \%$ when compared with titanium rods. ${ }^{6,9}$ However, in another biomechanical study that compared titanium rods, PEEK rods, and a dynamic stabilization device, it was reported that anterior load sharing was $55 \%, 59 \%$, and $75 \%$, respectively. ${ }^{12}$ This indicated that PEEK rods are closer to metal rods than truly dynamic devices for anterior load sharing. The endurance limit of a PEEK rod construct was similar to that of clinically useful metal systems. ${ }^{9}$ PEEK rods resulted in no increase in postfatigue motion compared with titanium rods in a biomechanical model. ${ }^{13}$ Intradiscal pressures at PEEK instrumented segments were similar to uninstrumented segments and greater than those with titanium rod constructs. ${ }^{14}$ Intradiscal pressures at adjacent segments were highest with dynamic devices, intermediate with semirigid rods, and lowest with rigid constructs; however, stress values at adjacent segments were lower in PEEK than titanium constructs in any direction of motion. ${ }^{15,16}$

\section{Fusion Rates}

The use of PEEK rods in lumbar fusion has been reported previously. 3,4,17,18 However, these studies featured small sample sizes, short follow up times, and contradictory results. ${ }^{4}$ Of 8 outcome reports found in a systematic review, 2 studies reported on procedures designed to create nonfusion outcomes (a third similar trial from 2013 was not included in the systematic review), and 1 study reported only on the condition of PEEK rods removed at subsequent surgery. ${ }^{3,19-21} \mathrm{Re}$ ported fusion rates varied from 86 to $100 \%$.

In 42 patients with PEEK rod fusions who were followed for a mean of 31.4 months, 5 patients required adjacent segment surgery and 3 patients were treated for interbody cage migration and nonunion. ${ }^{17}$ Radiographic fusion rate was $86 \%$. These authors concluded that PEEK rod fusion results were similar to those of other constructs, but not better, or perhaps worse than, metal rods. 
Other studies have reported better results with PEEK. 11,18,19,22-24 Highsmith and colleagues reported on 3 successful example cases of the use of PEEK rods. ${ }^{11}$ De lure and colleagues reported on 30 cases up to 5 levels (mean, 2.9) using autograft bone, with a mean follow up of 18 months. ${ }^{23}$ Results were reported as satisfactory. Three patients had radiographic nonunions, 1 of which required revision for asymptomatic screw loosening at the cranial end of the construct. Qi and colleagues, reported on 20 patients with PEEK rods compared to 21 patients with titanium alloy rods. ${ }^{24}$ Both groups had similar clinical outcomes, structural parameters, and $100 \%$ fusion rates. Athanasakopoulos and colleagues reported on 52 patients with up to 3 level fusions followed for a mean of 3 years. ${ }^{22}$ There were significant improvements in PROs: at 1 year $96 \%$ had radiographic union. Two patients had screw breakage, 1 of whom required revision to a metal rod construct. Colangeli and colleagues reported on 12 patients treated with PEEK rods compared with 12 who were treated with a dynamic system. ${ }^{18}$ They reported significant improvements, no complications, and $100 \%$ fusion at 6 months. Huang and colleagues reported on 38 patients intended to undergo a nonfusion procedure with 2 years of follow up. ${ }^{19}$ They reported good outcomes and 1 case of screw loosening. As no fusion was intended, no fusion outcomes were reported. All these studies suggested that longer follow up and more patients would be needed to assess the role of PEEK rods in lumbar fusion. ${ }^{3}$

Our results show a radiographic fusion rate of $86.4 \%$ and a radiographic nonunion rate of $9.9 \%$ in patients followed for at least 12 months. There was no clinical need for revision fusion at the index level. In our retrospective review, patients had high levels of smoking, DM, depression, immunosuppression, and obesity, which may negatively influence radiographic fusion rates when compared with other studies with $100 \%$ reported fusion rates. There was no instance of construct breakage or screw breakout, indicating that PEEK rods may allow enough flexibility to avoid construct failure under stress as in a fall.

\section{Patient Reported Outcomes}

Recent large studies were reviewed to assess the pre- and postoperative patient PROs reported in comparison with our study population (Table 4). In the Swedish Spine Registry analysis
TABLE 4 Study Score Comparisons ${ }^{25,26}$

\begin{tabular}{lcc} 
Studies & Preoperative & Postoperative, at 1 y \\
\hline Swedish 2017, mean (95\% Cl) & & \\
SF-36 physical component & $37.0(36-38)$ & $46.0(44-47)$ \\
ODI & $41.0(40-43)$ & $21.0(19-23)$ \\
\hline SPORT 2009, mean (SD) & & \\
SF-36 physical function & $30.4(21.9)$ & $58.5(1.6)$ \\
ODI & $45.5(16.9)$ & $19.2(1.3)$ \\
\hline This study, mean (SD) & & \\
SF-36 physical function & $28.9(19.0)$ & $59.3(21.3)$ \\
ODI & $24.8(7.9)$ & $14.5(7.6)$
\end{tabular}

Abbreviations: ODI, Oswestry Disability Index; SF-36, short form-36; SPORT, Spine Patient Outcomes Research Trial.

aPosterolateral fusion with instrumentation group.

${ }^{b}$ Posterolateral instrumented fusion with pedicle screws group.

of 765 patients with 3 different types of lumbar fusion, the mean preoperative ODI score was 37 and mean SF-36 physical component score (PCS) was 35 for the most similar approach (posterolateral fusion with instrumentation). ${ }^{25}$ At 1 year postoperation, the mean ODI was 26 and mean SF-36 PCS was 43. In the Spine Patient Outcomes Research Trial (SPORT) spondylolisthesis trial of 3 fusion types, the mean preoperative ODI was 41.2 and mean SF-36 PF score was 31.2 for the most similar approach (posterolateral instrumented fusion with pedicle screws). ${ }^{26}$ Postoperative ODI scores at 1 year decreased by a mean 20.9 points and mean SF-36 PF scores increased by 29.9 .

We report a mean preoperative SF-36 PF score of 28.9, which is lower than the SPORT study score for posterolateral fusion with instrumentation and the Swedish Study score for posterolateral instrumented fusion with pedicle screws. Similarly, our mean ODI score of 24.8 was better than the scores reported in the Swedish and SPORT studies. Our mean SF-36 PF score at 1 year postoperation was 59.3, compared with 58.5 for the SPORT study group and 46.0 in the Swedish study group. Mean ODI score at 1 year postoperatively was 14.5 , which is better than the scores reported in the Swedish and SPORT studies.

Minimally clinically important difference (MCID) is a parameter used to gauge the efficacy of spine surgery. The utility of the MCID based upon PROs has been questioned in lumbar fusion surgery, as it has been thought to measure if the patient is "feeling" rather than "doing" better, the latter of which can be 
better measured by functional performance measures and objective, external socioeconomic anchors such as return to work and health care costs. ${ }^{27}$ Nevertheless, validated PROs are reported widely in the spine surgery literature. The MCID in the SF-36 is not well established and can depend upon whether the scores are at the extremes or more in the central range and whether there is large variability in the scores. ${ }^{28}$ Rheumatoid arthritis was estimated to be 7.1 points on the PF scale and 7.2 on the physical component summary (PCS). ${ }^{29}$ For total knee replacement, it has been estimated to be 10 points on the SF-36 PCS. ${ }^{30}$ Lumbar surgery was estimated to be 4.9 points for the SF-36 PCS and 12.8 points for the ODI. ${ }^{31}$ And the SPORT trial it has been estimated that a $30 \%$ change in the possible gain (or loss) may be an appropriate criterion. ${ }^{28}$

With a preoperative mean SF-36 PF of 28.9, a $30 \%$ improvement in the available range (70.1) would be 21 points, making our data mean improvement of 30 points above the MCID. With a mean preoperative ODI of 24.6, a $30 \%$ improvement in the available range (25.4) would be 7.6 points, making our data mean improvement of 10.3 points better than the MCID. Therefore, our outcome results are comparable with other lumbar fusion outcome studies in terms of degree of disability prior to surgery and amount of improvement from surgery.

\section{Adjacent Segment Disease}

The precise factors resulting in adjacent segment disease are not fully defined. ${ }^{3,32}$ In reviews of lumbar adjacent segment disease, reported rates ranged from $2.5 \%$ at 1 year up to 80 to $100 \%$ at 10 years, with lower rates with noninstrumented fusions. ${ }^{4,32-34}$ Annual incidence of symptomatic adjacent segment disease following lumbar fusion ranges from 0.6 to $3.9 \%$ per year. ${ }^{32,35,36}$ Mismatch between lumbar lordosis and pelvic incidence after fusion is thought to lead to higher rates of adjacent segment disease, as can a laminectomy at an adjacent segment. ${ }^{32,36}$ Percutaneous fusion techniques or use of the Wiltse approach may lower the risk of adjacent segment disease due to avoidance of facet capsule disruption. ${ }^{37,38}$

Dynamic stabilization techniques do not appear be clearly protective against adjacent segment disease, although biomechanical models suggest that they may do so. ${ }^{33,39,40} \mathrm{~A}$ review by
Wang and colleagues pooled studies to assess the risk of lumbar adjacent segment disease in spinal fusion to compare to disc arthroplasty and concluded that fusion carried a higher risk of adjacent segment disease. ${ }^{41}$ Definitive data on other types of motion preservation devices is lacking. ${ }^{3}$

We show 3 adjacent segment fusions and 1 laminectomy have been needed in 108 patients and at a mean of 46 months after the index procedure and over 2.5 years of mean overall follow up. This is a low adjacent segment surgery rate compared to the historical data cited above, and may suggest some advantage for $\mathrm{PEEK}$ rods over more rigid constructs.

\section{Strengths and Limitations}

Strengths of this study include larger numbers than prior series of PEEK rod use and use in a population with high comorbidities linked to poor results without reduction in good outcomes. PEEK rods as used at the VAPHCS do not result in higher instrumentation costs than all metal constructs.

Study limitations include the retrospective nature with loss of follow up on some patients and incomplete radiographic and PROs in some patients. The use of $100 \%$ stereotactic guidance, the avoidance of interbody devices, and the offlabel use of bone morphogenetic protein as part of the fusion construct introduce additional variables that may influence comparison to other studies. To avoid unnecessary radiation exposure, flexion extension films or CT scans were not routinely obtained if patients were doing well. ${ }^{42}$ Additionally, the degree of motion on dynamic views that would differentiate pseudarthrosis from arthrodesis has not been defined. ${ }^{5}$

\section{CONCLUSIONS}

The results presented show that lumbar fusion with PEEK rods can be undertaken with short hospitalization times and low complication rates, produce satisfactory clinical improvements, and result in radiographic fusion rates similar to metal constructs. Low rates of hardware failure or need for revision surgery were found. Preliminarily results of low rates of adjacent segment surgery are comparable with previously published metal construct rates. Longer follow up is needed to confirm these findings and to investigate whether semirigid constructs truly offer some protection from adjacent segment disease when compared to all metal constructs. 
Acknowledgments

The authors thank Shirley McCartney, PhD, for editorial assistance.

\section{Author disclosures}

The authors report no actual or potential conflicts of interest with regard to this article.

\section{Disclaimer}

The opinions expressed herein are those of the authors and do not necessarily reflect those of Federal Practitioner, Frontline Medical Communications Inc., the US Government, or any of its agencies.

\section{References}

1. Deyo RA, Mirza SK, Martin BI, Kreuter W, Goodman DC, Jarvik JG. Trends, major medical complications, and charges associated with surgery for lumbar spinal stenosis in older adults. JAMA. 2010;303(13):1259-1265. doi:10.1001/jama.2010.338

2. Machado GC, Maher CG, Ferreira PH, et al. Trends, complications, and costs for hospital admission and surgery for lumbar spinal stenosis. Spine (Phila Pa 1976). 2017;42(22):1737-1743. doi:10.1097/BRS.0000000000002207

3. Li C, Liu L, Shi JY, Yan KZ, Shen WZ, Yang ZR. Clinical and biomechanical researches of polyetheretherketone (PEEK) rods for semi-rigid lumbar fusion: a systematic review. Neurosurg Rev. 2018;41(2):375-389. doi:10.1007/s10143-016-0763-2

4. Mavrogenis AF, Vottis C, Triantafyllopoulos G, Papagelopoulos PJ, Pneumaticos SG. PEEK rod systems for the spine. Eur J Orthop Surg Traumatol. 2014;24 Suppl 1:S111-S116. doi:10.1007/s00590-014-1421-4

5. Choudhri TF, Mummaneni PV, Dhall SS, et al. Guideline update for the performance of fusion procedures for degenerative disease of the lumbar spine. Part 4: radiographic assessment of fusion status. J Neurosurg Spine. 2014;21(1):23-30. doi:10.3171/2014.4.SPINE14267

6. Ahn YH, Chen WM, Lee KY, Park KW, Lee SJ. Comparison of the load-sharing characteristics between pediclebased dynamic and rigid rod devices. Biomed Mater. 2008;3(4):044101. doi:10.1088/1748-6041/3/4/044101

7. Ozer AF, Cevik OM, Erbulut DU, et al. A novel modular dynamic stabilization system for the treatment of degenerative spinal pathologies. Turk Neurosurg. 2019;29(1):115120. doi:10.5137/1019-5149.JTN.23227-18.1

8. Hak DJ, Mauffrey C, Seligson D, Lindeque B. Use of carbon-fiber-reinforced composite implants in orthopedic surgery. Orthopedics. 2014;37(12):825-830. doi:10.3928/01477447-20141124-05

9. Gornet MF, Chan FW, Coleman JC, et al. Biomechanical assessment of a PEEK rod system for semi-rigid fixation of lumbar fusion constructs. J Biomech Eng. 2011;133(8):081009. doi:10.1115/1.4004862

10. Jackson JB 3rd, Crimaldi AJ, Peindl R, Norton HJ, Anderson WE, Patt JC. Effect of polyether ether ketone on therapeutic radiation to the spine: a pilot study. Spine (Phila Pa 1976). 2017;42(1):E1-E7. doi:10.1097/BRS.0000000000001695

11. Highsmith JM, Tumialán LM, Rodts GE Jr. Flexible rods and the case for dynamic stabilization. Neurosurg Focus. 2007;22(1):E11. Published 2007 Jan 15. doi:10.3171/foc.2007.22.1.11

12. Sengupta DK, Bucklen B, McAfee PC, Nichols J, Angara $\mathrm{R}$, Khalil $\mathrm{S}$. The comprehensive biomechanics and loadsharing of semirigid PEEK and semirigid posterior dynamic stabilization systems. Adv Orthop. 2013;2013:745610. doi:10.1155/2013/745610

13. Agarwal A, Ingels M, Kodigudla M, Momeni N, Goel V, Agarwal AK. Adjacent-level hypermobility and instrumented-level fatigue loosening with titanium and PEEK rods for a pedicle screw system: an in vitro study. J Biomech Eng. 2016;138(5):051004. doi:10.1115/1.4032965
14. Chou WK, Chien A, Wang JL. Biomechanical analysis between PEEK and titanium screwrods spinal construct subjected to fatigue loading. J Spinal Disord Tech. 2015;28(3):E121-E125. doi:10.1097/BSD.0000000000000176

15. Shih KS Hsu CC, Zhou SY, Hou SM. Biomechanical investigation of pedicle screw-based posterior stabilization systems for the treatment of lumbar degenerative disc disease using finite element analyses. Biomed Eng: Appl Basis Commun. 2015;27(06):1550060. doi: 10.4015/S101623721550060X

16. Chang TK, Huang $\mathrm{CH}$, Liu YC, et al. Biomechanical evaluation and comparison of polyetheretherketone rod system to traditional titanium rod fixation on adjacent levels. Formosan J Musculoskeletal Disord. 2013;4(2):42-47. doi: 10.1016/j.fjmd.2013.04.003

17. Ormond DR, Albert L Jr, Das K. Polyetheretherketone (PEEK) rods in lumbar spine degenerative disease: a case series. Clin Spine Surg. 2016;29(7):E371-E375. doi:10.1097/BSD.0b013e318277cb9b

18. Colangeli $S$, Barbanti Brodàno $G$, Gasbarrini $A$, et al. Polyetheretherketone (PEEK) rods: short-term results in lumbar spine degenerative disease. J Neurosurg Sci. 2015;59(2):91-96

19. Huang W, Chang Z, Song R, Zhou K, Yu X. Non-fusion procedure using PEEK rod systems for lumbar degenerative diseases: clinical experience with a 2-year follow-up. BMC Musculoskelet Disord. 2016;17:53. Published 2016 Feb 1. doi:10.1186/s12891-016-0913-2

20. Wang C-J, Graf H, Wei H-W. Clinical outcomes of the dynamic lumbar pedicle screw-rod stabilization. Neurosurg Q. 2016;26(3):214-218. doi:10.1097/WNQ.0000000000000169

21. Kurtz SM, Lanman TH, Higgs G, et al. Retrieval analysis of PEEK rods for posterior fusion and motion preservation. Eur Spine J. 2013;22(12):2752-2759. doi:10.1007/s00586-013-2920-4

22. Athanasakopoulos M, Mavrogenis AF, Triantafyllopoulos G, Koufos S, Pneumaticos SG. Posterior spinal fusion using pedicle screws. Orthopedics. 2013;36(7):e951-e957. doi:10.3928/01477447-20130624-28

23. De lure F, Bosco G, Cappuccio M, Paderni S, Amendola $L$. Posterior lumbar fusion by peek rods in degenerative spine: preliminary report on 30 cases. Eur Spine J. 2012;21 Suppl 1(Suppl 1):S50-S54. doi:10.1007/s00586-012-2219-x

24. Qi L, Li M, Zhang S, Xue J, Si H. Comparative effectiveness of PEEK rods versus titanium alloy rods in lumbar fusion: a preliminary report. Acta Neurochir (Wien). 2013;155(7):1187-1193. doi:10.1007/s00701-013-1772-3

25. Endler P, Ekman P, Möller H, Gerdhem P. Outcomes of posterolateral fusion with and without instrumentation and of interbody fusion for isthmic spondylolisthesis: a prospective study. J Bone Joint Surg Am. 2017;99(9):743-752. doi:10.2106/JBJS.16.00679

26. Abdu WA, Lurie JD, Spratt KF, et al. Degenerative spondylolisthesis: does fusion method influence outcome? Four-year results of the spine patient outcomes research trial. Spine (Phila Pa 1976). 2009;34(21):2351-2360. doi:10.1097/BRS.0b013e3181b8a829

27. Gatchel RJ, Mayer TG, Chou R. What does/should the minimum clinically important difference measure? A reconsideration of its clinical value in evaluating efficacy of lumbar fusion surgery. Clin J Pain. 2012;28(5):387-397. doi:10.1097/AJP.0b013e3182327f20

28. Spratt KF. Patient-level minimal clinically important difference based on clinical judgment and minimally detectable measurement difference: a rationale for the SF-36 physical function scale in the SPORT intervertebral disc herniation cohort. Spine (Phila Pa 1976). 2009;34(16):1722-1731. doi:10.1097/BRS.0b013e3181a8faf2

29. Ward MM, Guthrie LC, Alba MI. Clinically important changes in short form 36 health survey scales for use in rheumatoid arthritis clinical trials: the impact of low responsiveness. Arthritis Care Res (Hoboken). 2014;66(12):17831789. doi:10.1002/acr.22392 
30. Escobar A, Quintana JM, Bilbao A, Aróstegui I, Lafuente I, Vidaurreta I. Responsiveness and clinically important differences for the WOMAC and SF-36 after total knee replacement. Osteoarthritis Cartilage. 2007;15(3):273-280. doi:10.1016/j.joca.2006.09.001

31. Copay AG, Glassman SD, Subach BR, Berven S, Schuler TC, Carreon LY. Minimum clinically important difference in lumbar spine surgery patients: a choice of methods using the Oswestry Disability Index, Medical Outcomes Study questionnaire Short Form 36, and pain scales. Spine J. 2008;8(6):968-974. doi:10.1016/j.spinee.2007.11.006

32. Radcliff KE, Kepler CK, Jakoi A, et al. Adjacent segment disease in the lumbar spine following different treatment interventions. Spine J. 2013;13(10):1339-1349. doi:10.1016/j.spinee.2013.03.020

33. Epstein NE. Adjacent level disease following lum bar spine surgery: a review. Surg Neurol Int 2015;6(Suppl 24):S591-S599. Published 2015 Nov 25. doi:10.4103/2152-7806.170432

34. Epstein NE. A review: reduced reoperation rate for multilevel lumbar laminectomies with noninstrumented versus instrumented fusions. Surg Neurol Int 2016;7(Suppl 13):S337-S346. Published 2016 May 17. doi:10.4103/2152-7806.182546

35. Scemama C, Magrino B, Gillet P, Guigui P. Risk of adjacent-segment disease requiring surgery after shor lumbar fusion: results of the French Spine Surgery Society Series. J Neurosurg Spine. 2016;25(1):46-51. doi:10.3171/2015.11.SPINE15700

36. Tempel ZJ, Gandhoke GS, Bolinger BD, et al. The influence of pelvic incidence and lumbar lordosis mismatch on development of symptomatic adjacent leve disease following single-level transforaminal lumbar interbody fusion. Neurosurgery. 2017;80(6):880-886. doi:10.1093/neuros/nyw073

37. Cheng YW, Chang PY, Wu JC, et al. Letter to the editor: Pedicle screw-based dynamic stabilization and adjacentsegment disease. J Neurosurg Spine. 2017;26(3):405-406. doi:10.3171/2016.7.SPINE16816

38. Street JT, Andrew Glennie R, Dea N, et al. A comparison of the Wiltse versus midline approaches in degenerative conditions of the lumbar spine. J Neurosurg Spine. 2016;25(3):332-338. doi:10.3171/2016.2.SPINE151018

39. Kuo CH, Huang WC, Wu JC, et al. Radiological adjacentsegment degeneration in L4-5 spondylolisthesis: comparison between dynamic stabilization and minimally invasive transforaminal lumbar interbody fusion. J Neurosurg Spine. 2018;29(3):250-258. doi:10.3171/2018.1.SPINE17993

40. Lee CH, Kim YE, Lee HJ, Kim DG, Kim CH. Biomechanical effects of hybrid stabilization on the risk of proximal adjacent-segment degeneration following lumbar spinal fusion using an interspinous device or a pedicle screw-based dynamic fixator. J Neurosurg Spine. 2017;27(6):643-649. doi:10.3171/2017.3.SPINE161169

41. Wang JC, Arnold PM, Hermsmeyer JT, Norvell DC. Do lumbar motion preserving devices reduce the risk of adjacent segment pathology compared with fusion surgery? A systematic review. Spine (Phila Pa 1976). 2012;37(22 Suppl):S133-S143. doi:10.1097/BRS.0b013e31826cadf2

42. Ross DA. Letter to the editor: steroid use in anterior cervical discectomy and fusion. J Neurosurg Spine. 2016;24(6):998-1000. doi:10.3171/2015.9.SPINE151052 